\title{
Efficacy of Rituximab in the treatment of pemphigus: experience of Moroccan population
}

\section{Hanane Bay Bay, Ghita Senhaji, Sara Elloudi, Zakia Douhi, Fatima Zahra Mernissi}

\author{
Department of Dermatology, UHC Hassan II, Fez, Morocco
}

Corresponding author: Dr. Hanane Bay Bay, E-mail: hananebaybay@gmail.com

\begin{abstract}
Background: Pemphigus is a severe autoimmune blistering disease mediated by pathogenic anti-desmoglein antibodies leading to an inter keratinocyte disjunction. Rituximab is a monoclonal antibody that binds to the CD-20 antigen of B lymphocytes, which causes B-cell depletion and a subsequent reduction in pathogenic autoantibodies. Its therapeutic role in vulgaris and superficial pemphigus has been progressively growing with increasing evidence of successful outcomes. Material and Methods: It was a prospective study extending for two years in the dermatology department of the UHC Hassan II in Fez, dealing with patients with pemphigus who are severe or resistant to conventional treatments treated with Rituximab. We analyzed the efficacy and tolerance of Rituximab in the treatment of severe and refractory forms of our pemphigus population. Results: Patients treated by Rituximab were 13. Eleven cases of pemphigus vulgaris and two cases of superficial pemphigus In our series, Rituximab was used with satisfactory, indeed, 6 patients achieved complete remission, while 4 had partial remission. Side effects of Rituximab were infectious complications. Conclusions: We highlight the efficiency and safety of Rituximab in our series.
\end{abstract}

Key words: Vulgaris pemphigus; Superficial pemphigus; Morocco; Herpetic infection

\section{INTRODUCTION}

Pemphigus is a severe autoimmune blistering disease mediated by pathogenic anti-desmoglein antibodies leading to an inter keratinocyte disjunction [1,2]. Rituximab is a monoclonal antibody that binds to the CD-20 antigen of B lymphocytes, which causes B-cell depletion and a subsequent reduction in pathogenic autoantibodies $[1,2]$. Its therapeutic role in pemphigus has been progressively growing with increasing evidence of successful outcomes. It's an emerging therapy for severe pemphigus vulgaris and superficial pemphigus [1-4].

\section{Objective}

Our aim was to analyze the efficacy and tolerance of Rituximab in the treatment of severe and refractory forms of our pemphigus population.

\section{MATERIAL AND METHODS}

This is a prospective study extending from 2016 to 2018, in the dermatology department of the CHU Hassan II in Fez, dealing with patients with pemphigus who are severe or resistant to conventional treatments treated with Rituximab. Therapeutic response, duration of remission, therapeutic tolerance and side effects were assessed. All patients received a course of Rituximab at a dose of $375 \mathrm{mg} / \mathrm{m} 2 /$ week for 4 weeks combined with oral corticosteroid therapy at a dose of $0.5 \mathrm{mg} / \mathrm{kg} /$ day.

\section{RESULTS}

Patients treated by Rituximab were 13. The average age was 55 with extremes ranging from 34 to 75 years, with a clear female predominance with a percentage of $84 \%$. There were 11 cases of pemphigus vulgaris and 2 cases of superficial pemphigus. Nikolski's sign was positive

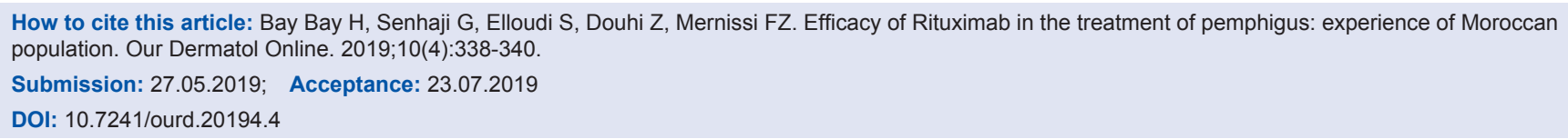


in 8 patients. Cutaneous involvement was present in 11 patients. Mucosal involvement was found in 8 patients with erosions. Most patients had severe disease. All our patients had histological confirmation and IFD of their pathology. IFI was very high in all patients.

3 patients were initially treated by Rituximab while 10 others received their Rituximab treatment after failure of other therapies which were oral corticosteroid alone in 1 patient, associated with Azathioprine in 7 patients alone or followed by cyclophosphamide bolus in 3 patients or associated with DDS in 2 patients.

The average duration of follow-up was 6 months. The use of Rituximab was associated with a significant reduction in corticosteroid dose during followup. After 3 months, 6 patients achieved complete remission (Figs. 1 and 2), while 4 had partial remission (Figs. 3 and 4) with persistent endobuccal erosions. 2 patients had a relapse of their disease after 6 months

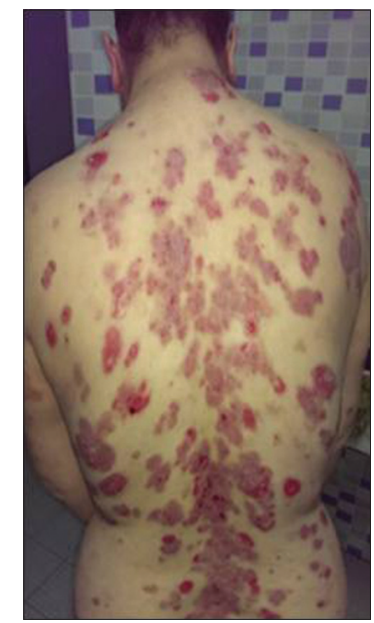

Figure 1: Vulgaris pemphigus before treatment.

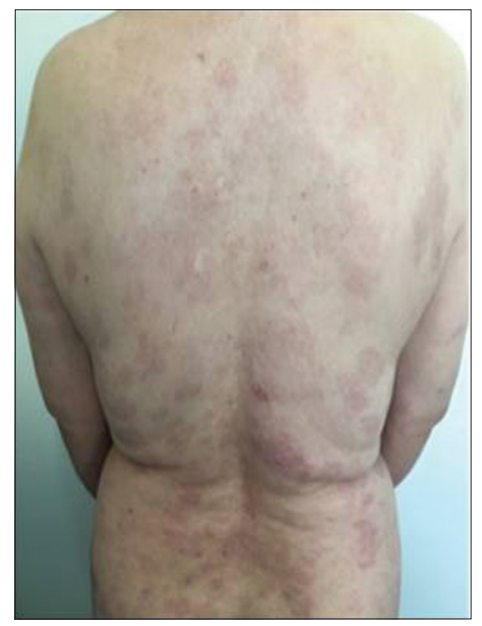

Figure 2: The same patient after 6 months of Rituximab. of treatment. The relapses were taken care of by a new cure of Rituximab. The control IFI after 6 months had significantly decreased in all patients. No case of death was encountered.

Side effects of Rituximab were seen in 7 patients with delayed and infectious complications. 3 patients had bacterial and urinary cutaneous co-infection, 2 had had herpetic superinfections, while 1 patient had an episode of erysipelas.

\section{DISCUSSION}

Our study highlights the efficacy of Rituximab in treating severe and refractory forms of pemphigus to other treatments. Which joins the different studies already published [1-4].

We also demonstrate the interest of this molecule as a first-line treatment in our Moroccan context where

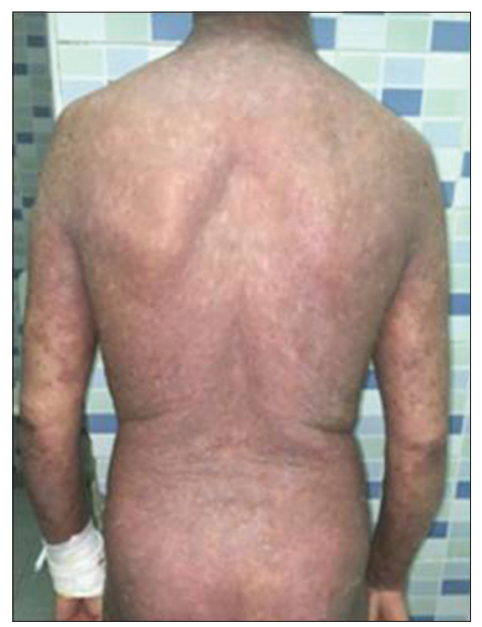

Figure 3: Superficial pemphigus before treatment.

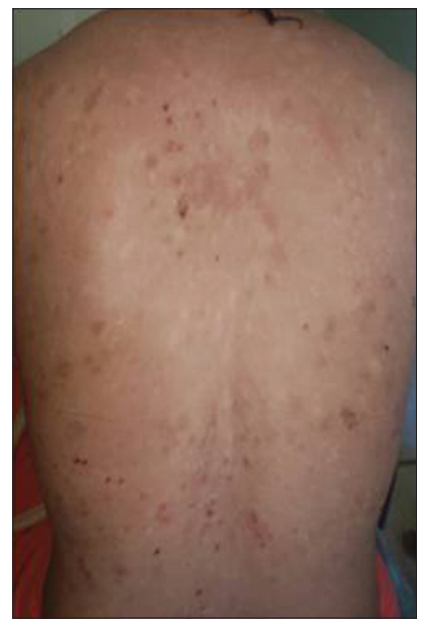

Figure 4: Superficial pemphigus after one month of Rituximab. 
severe and extensive forms predominate. This is in perfect concordance with the new data from the literature. Use of Rituximab was beneficial in $77 \%$ of patients, leading to faster clinical improvement and longer long-term remission. This in patients who have already received conventional treatment with corticosteroids and immunosuppressants with therapeutic failure. The decline will allow us to assess whether the remissions will be extended. All cases demonstrated significant improvement or complete remission and most experienced no adverse events [4-8] Rituximab appears to be both well tolerated and efficacious for refractory juvenile pemphigus foliaceus [6]. Therefore, it may be considered for severe cases of $\mathrm{PF}$ to avoid side effects associated with conventional glucocorticoid therapy [5,7-10]. This study reinforces the beneficial role of rituximab in pemphigus in our context. Pemphigus foliaceus patients required a higher total cumulative dose of prednisolone over a longer time to achieve remission and the remission lasted longer than that in pemphigus vulgaris $[5,11,12]$.

\section{CONCLUSIONS}

Treatment with Rituximab is a safe and effective method for the treatment of severe forms of pemphigus and refractory to other treatments in our series. It allows rapid control with cortisone sparing. The main obstacle lies in the cost of this treatment and its support pending marketing authorization in Morocco.

\section{ACKNOWLEDGEMENTS}

We are indebted to all patients who participated in this study and gave their consent. We thank all volunteer investigators and the medical staff of the Department of Dermatology, and clinical epidemiology for their enormous help.

\section{Statement of Human and Animal Rights}

All procedures followed were in accordance with the ethical standards of the responsible committee on human experimentation (institutional and national) and with the Helsinki Declaration of 1975, as revised in 2008.

\section{Statement of Informed Consent}

Informed consent was obtained from all patients for being included in the study.

\section{REFERENCES}

1. Ahmed AR, Shetty S. A comprehensive analysis of treatment outcomes in patients with pemphigus vulgaris treated with rituximab. Autoimmun Rev. 2015;14:323-31.

2. Loh TY, Paravar T. Rituximab in the management of juvenile pemphigus foliaceus. Dermatol Online J. 2017; 23(6). pii:13030/qt0415n1r5.

3. Hebert V, Joly P. Rituximab in pemphigus. Immunotherapy. 2018;10:27-37.

4. Baum S, Gilboa S, Greenberger S, Pavlotsky F, Trau H, Barzilai A. Adjuvant rituximab therapy in pemphigus: a single-center experience of 18 cases. J Dermatol Treat. 2013;24:427-30.

5. De D, Bishnoi A, Handa S, Mahapatra T, Mahajan R. Effectiveness and safety analysis of rituximab in 146 Indian pemphigus patients: A retrospective single-center review of up to 68 months follow-up. Indian J Dermatol Venereol Leprol. 2019 May 9. doi: 10.4103/ijdvl. IJDVL_848_17. [Epub ahead of print]

6. Arduino PG, Broccoletti R, Carbone M, Gambino A, Sciannameo V, Conrotto D, et al. Long-term evaluation of pemphigus vulgaris: A retrospective consideration of 98 patients treated in an oral medicine unit in north-west Italy. J Oral Pathol Med. 2019; 48: 406-12.

7. Toosi R, Mahmoudi H, Balighi K, Teimourpour A, Alaeen H, Shaghaghi M, et al. Efficacy and Safety of Biosimilar Rituximab in Patients with Pemphigus Vulgaris: A Prospective Observational Study. J Dermatolog Treat. 201910:1-28.

8. Patsatsi A, Kyriakou A, Douma S, Kokolios M, Meltzanidou P, Lambropoulos A, et al. Non-endemic erythrodermic pemphigus foliaceus: a case with delayed diagnosis and response to rituximab. J Dtsch Dermatol Ges. 2019; 17: 537-9.

9. Kurihara Y, Yamagami J, Funakoshi T, Ishii M, Miyamoto J, Fujio Y, et al. Rituximab therapy for refractory autoimmune bullous diseases: A multicenter, open-label, single-arm, phase $1 / 2$ study on 10 Japanese patients. J Dermatol. 2019; 46:124-30.

10. Kasperkiewicz M, Shimanovich I, Ludwig RJ, Rose C, Zillikens D, Schmidt E. Rituximab for treatment-refractory pemphigus and pemphigoid: a case series of 17 patients. J Am Acad Dermatol. 2011;65:552-8.

11. Kim MR, Kim HC, Kim SC. Long-term prognosis of pemphigus in Korea: retrospective analysis of 199 patients. Dermatology. 2011;223:182-8.

12. Nigam R, Levitt J. J Drugs Dermatol. Where does rituximab fit in the treatment of autoimmune mucocutaneous blistering skin disease? 2012;11:622-5.

Copyright by Hanane Bay Bay, et al. This is an open-access article distributed under the terms of the Creative Commons Attribution License, which permits unrestricted use, distribution, and reproduction in any medium, provided the original author and source are credited.

Source of Support: Nil, Conflict of Interest: None declared. 